

\section{Obras firmadas de Cristóbal Lozano para los jesuitas}

Ricardo Estobridis tandenas

tiniversided Kacione Maror Af San Marces

El articulo se centra en el analisis de dos nuevas obras del pintor limeño paradiemático del siglo Xvil, Cristóbal Lozano, ubicadas en la Iffesia de san Pedro de Uima, donde se representan dos escenas de la vida de San Luis Gonzaga. Estas obres tempranas se suman a otra obra importante realizada por el pintor para la ordon jesuita, rocientemente restaurada. Asimismo, se realiza la identificación de un posible autorretrato del artista limeño $x$ de una pintura existente en la Catedral de Lima, que se considera de este autor y es punto de estudio para el desarrollo parcial de su fuente grabada en muchas de sus obras posteriores,

\section{Cristobal Loaano / Portaretrato / Arte peruano / Pintura virreinal / Orden jesuita}

This article focuses on the anaiysis of two newworks by the paradigmatic eighteenth-century painter from lima, Cristobal Lozana. These two works, Iocated at San Pedro church in Lima, depict two scenes from the life of Saint Aloysius Gonzaga. These early works rest along another important recently nestorod work by the artist for the Jesuit order. Furthermoce, a possible self-portrait of the artist and a painting in the Cathedral of tima aro under authentication. This latter is the subject of study for the partial development of his engraved sources in many of his later works.

Cristóbal Lozano / Portrait / Peruvian art / Peruvian viceroyalty painting / Jesuit order

Hace poco más de dos lustros, en el 2001, presentamos en el III Congreso Internacional del Barroco Jberoamericano llevado a cabo en la ciudad de 5evilla un trabajo de investigación sobre el pintor limeño Cristóbal Lozano, que fue la culminación de varias notas y ensayos anteriores. Ponencia que fue publicada en Fspaña (Fstabridis 2001 y y comentada generoSamente por el historiadot Antonio San Cristóbal en la Revista Atrio de la Universidad Pablo de Olavide de Sevilla:

El trabajo de Fscabridis Cardenas es un boillante modelo de exposición historica sobre la figura del pintor Cristóbal Lozano. El autor nos ofrece una visiós integral de la Łpoca y del pintor, como resultado de las nume-

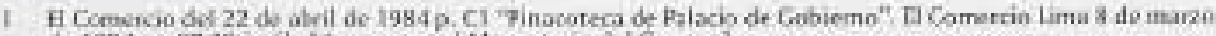

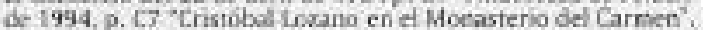

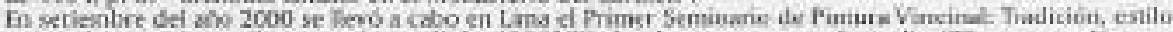

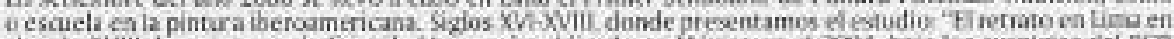

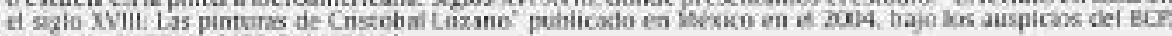

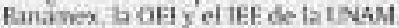


10sas. y diriamos exhaustivas, investigaciones sobre los cuadros pintados por Lozans y también acerca del centexto social en que surgieran?

Aun asf consideramos que quedaba mucho camino por andar y persistimos en las investigacinnes subre este insigne maestro para ponerlo en sa justo valor en la historia del arte peruano y aos satisface que colegas como Luis Eduando Wuffarden y sobre todo nuestro ex alumno de la Escuela de Arte de la Uaiversidad de San Marcos. Ricardo Kusunoki, hayan realizado después de auestra publicación importantes aportes a los estudios sobre Lozano yel contexto de su época?

Fin el presente estudio daremos a la luz dos obras inoditas de Laxano cque se conservan en la iglesia de San Pedro de Lima, firmadas por su autoc en 1730, la fecha más temprana con. signada pot el pintor en su periplo artístico limeno y que marcaría una relación con la orden jesuita en fechas iniciales de su ptoducción, cuando tendria unos 25 ahos y mo era aún conocido en la ciudod donde tiempo despues, en sas anos de madarez, seria comparado con Rafael y Miguel Angeli, retrataria a virneyes, catedraticos de San Maccos y a la nobleza limecia e incluso crearia una obra singular para ser obsequiada a Carlos 3II. rey de Espanta?,

La etapa temprana de Lozano ya la hemos definido desde que diéramos a conocer ten 1994 la Sere de lo Viclo de Ja Virgen, conservadz en el Monasterio del Carmen de Lima, obra fimada con la humildad de un principiante; "Por amor de Diøs un Ave Maria por Lozano", pero sin fecha, y la lmposicián de la casilla a San iddefonso, firmada con la misma modestia: "Dionoslo Christóval Lozano quien lo piato", firmada en 1734. A ellas debermos sumar aho. ra las pinturas que se conservan en la Capila de San Luis Gonzage de la iglesia de San Pedro de Lima, donde se representan dos escenas importantes de la vida del joven santo jesaita, patrón de la jeventud, desarrolladas con el mismo estilo temprano donde la influencia de la pintura cusqueña que por entonces inundaba el mercado local es evidente. Asimismo. las pinturas levan la autoria discreta de firmar en uno de los fienzos, en la parte posterior: "Mro. Lozano. Un amigo" y en el otro en la parte anterion: - Lima, A, 1730 ".

En la iglesia jesuita de Lima no podia faltar una capilla dedicada a San luis Gonzaga y es así que se destina para su culto la segunda del lado de la epistola. Esto debió suceder hacia 1640-41 ya que el padre Vargas Ugarte', es base a una Mernoria de escos anos, menciona varios retablos que son de esta época. entre ellos el retablo de San luis. Gonzaga, aunque por las características que presenta el actual, corresponde a una fecha posterior. La importancia del retablo fue destacada al colocar en el la copia de la pintura que San Lucas hizo de la Virgen, obsequiada a los jesuitas de lima por San Francisco de Borja?.

El retablo actual de Sen Luis Gonzaga dele corresponter a aquelos retablos tempranos que se hicieson en Lima denro del espiritu de un harmoco avanzado, los que ya incluyeroo entre sus elementos arquitectónicas h coltumna solomórica. Este elemento está estrechamente ligado al escultor vasco Diego de Aguime, activo en Lima entre 1667 y 1718 , a quien en 1675 se le encargó el retablo mayor para la Catedral, pruyecto crunce que iralsguraba la indusión de este tipo de columnas en la retablistica limena. Sin embargo, posteriormente las induina en 1681

$2 \sin$ Cistabi-2005:140

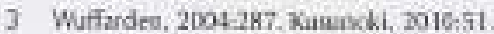

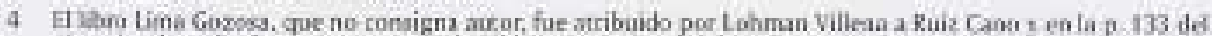

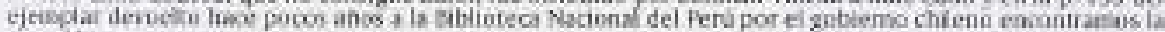
monoin.

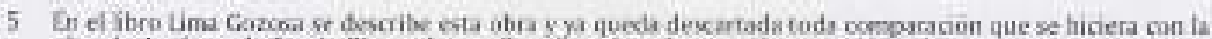

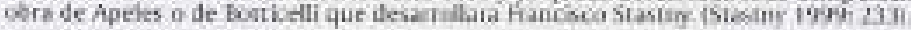

fi Wiryas, 106523

7 Capel 146933. 
en la capilla de las inimas de la igle. sia de San Marcelot. Les acbo reta blos salomónicos de San Pedro son anoinimos aun y de seguro fueron realizados pot estos anos det uiltimo cuarto de siglo XVII?

Complementando ta omamen. tacion del retablo de San Luis Gonzaga en 1730 se incluyeron en esta capilla dos lienzos de gran formato encargados a Cristobal Lozano, los que oman la parte superior de los muros laterales. Los temas iconograficos corresponden a los dos momentos más imporfantes de su vida, en el muro de ta ixquierda cuando la virgen de Buen Consejo le habla en una igle sia madrilena y en ol mum do la derecha ef modento que es recibido en el noviciado de la crden jesuita en Italia.

Luis Gonzaga es uno de los santos de la cristiandad que procede de ilustre prosapia italiana, hijo de Don Femando Gonzaga. principe del imperio y marques de Castiglinni, de la provincia de

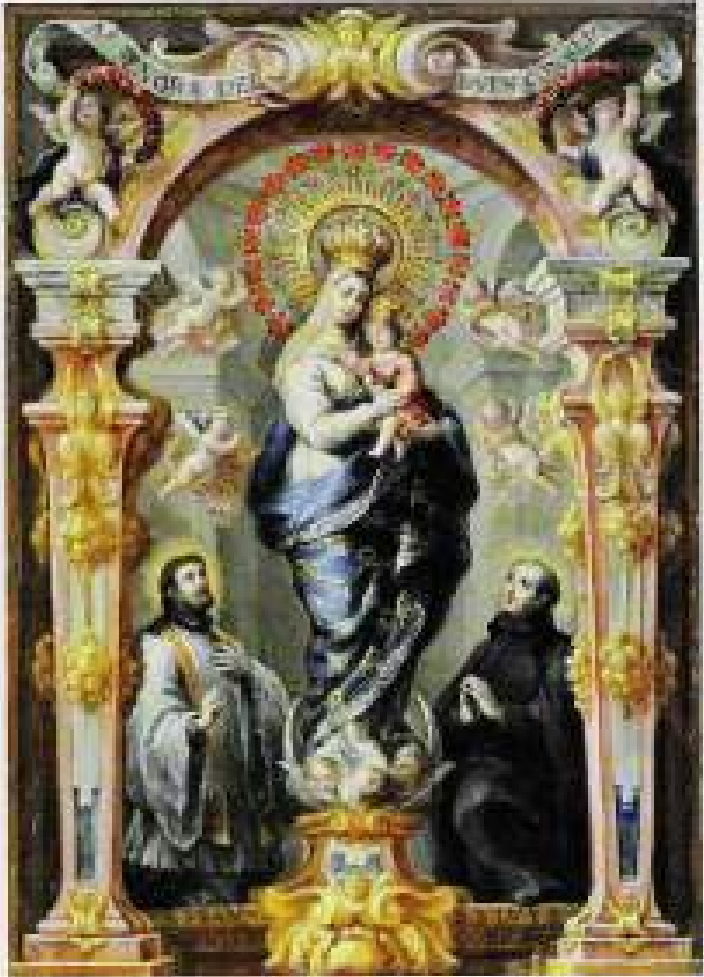

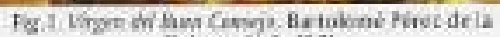
Detrese siglo $x$ ill Stivieri en Lombardia, y de Donิa María Tana Santena, natural de Chien en el Piamonte con quien se casó en Espana bajo la asuerxia de la reina lsabel de Kalois. esposa del rey Felipe Il, ya qut era una de sus damas. Después de la boda los marqueses volvicron a Italia y en ser fortaleza de Castiglioni nació su primogenito Luis el 9 de marzo de 1568, bautizado al poco tiempo ftwo como padnino al primo de su padre, Don Guillermo Duque de Mantua".

Desde la tempran edad de Luis la influencia de la religiosidad de su madre se dejó sentí en él, tanto que apenas con 8 ahos, en su estancia en Forencial, prometió vurginidad ante la Virgen Anunciada, cumplida hasta su muette:

Ein 1581, cuando luis contaba con 13 años, el viaje de Bohemia a Espata de la Emperatriz Maria de Austria, hija de Caslos V, mujer del Emperador Maximiliano ll y madre del Enperador reinante Rodolfo II se determino, por encargo de su hermano el rey felipe II, que fueta acoanpahada la eimperatriz por nobles italianos que tuvieran alguna dependenca de ia corona, por tanto, don Fernando y su esposa foeron ciegidos, trasladandose la fambia Conzaga a España. Ya en aquel reino, Luis y su hermano Rodolío fueron destinados a ser menimos def principe Diegcr, hio de Felipe II. A perar de su destine en la corte, Luis no se alcóo de s. deseo gestado en su mente desde Italia, la intención de encrar en algún momento a servir a Dios en alguna

\footnotetext{
6 Hat-iere, 1927;184,

9 Wiflerden 1996:46.

10 Cepari 1895 . Tom Prinero, Cap I
} 


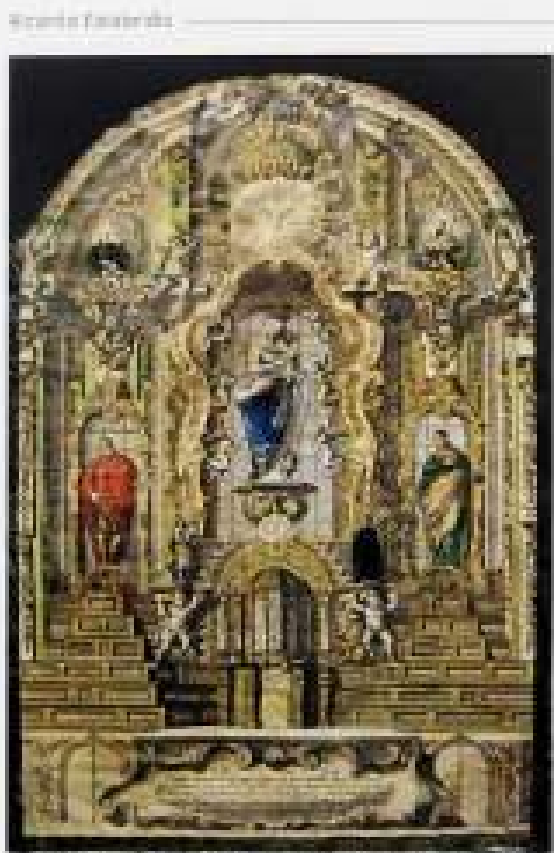

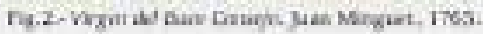

orden reliziosa, En un primer momento penso en varias órdenes, pero su deseo manifiesto de no pretender anguna dignidad edesiastica lo llewo a considerat que posdria ser la Companina de lesus, ya que en esta orden religiosa ese noto no era omitido y solo podia incumplise por onder papal, asi no seria obligado por su posicion y familia a aspirar a tales digndades"

Con la finalidad de no errar en su decerminacinon de entrar a la orden jestisa, puso csiso intercesora a la Virget para que ella le haga saber lir decisióa de Dios. Por ello, el dia de la Asunción de la Virgen del año de 1583, calando contaba quince anos de edad, despues de comalgar se retimo a orat ante una imagen de la Virgen con el Nino caya tradición remon ta batyocacios al siglo XV en el paeblo the Genazzano en Italia, conservada en la antizua Iglesia de San Pedro y San Pablo, edificada en Madrid bajo el patrocinis de la emperatriz Maria de Austria en 1560, donde se ubxicaria poseriormeate a Colegio Imperial de los jesuitas" la esculcura de la Virgen era de cuerpo entero, ralladia en madera y policromada.

diferente a la italiana que es en busto. H milagro ocurrió entonces y forma parte importante del momento principal de su biografia y por tanto fuente de inspiración para una de las representaciones pictóricas que realizá Lozano para su capila en Lima, cuando en étasís escucha la voz de la Virgen que le dice: "Entra en la Compañía de mi Hijo".

E pequeño templo madrileño en mencióa fue demolido en 1608 y en 1651 ya se erguia el aurevo templo y Colegio de la Compania, mucho más amplio, que inchuý terrenus ale, canos y se le llamo Imperial por estar bajo el patrocinio de la Emperatriz, dedicado a San Francisco Javiet. En este nuevo templo la fama de la actwocacion de la llamada Virgen del Baca Conscjo, lamada asi desde entonces, es manifiesta en la devoción del pueblo matdéleño de la época y en pinturas como la realizada sobre plancha de cobre por el artista lugareño Ilartolomé Perez de la Dehesa (1634-1698), conocido per sus pinturas de flores y decoraciones efimeras, obta adquirida filtimamente por el Museo de Arte de Cleveland (Fig. 1). Igualmente, en un grabado procedente del siglo XVII de Juan Miogoct, firmado en 1765 , donde la Virgen es representada en otro altar ya con caracteres ormamentales rococo, acompañada en las bormacinas laterales por San lsiciso y su esposa Santa Maria de la Cabeza. Estarpa de devocion que lleva en la zona inferior una leyenda:

\footnotetext{
Vo-Ro de Na. Sa, del Buen Consejo, llamada assi por eique con vaz sersible dio a San buis Gonzaga. Se venera en su capilla del Calegio Imperial de la Compania de IHS de esta corte. Son muchas las indulgencias concedidas a esta imazen y las que puedan ganarse por cada Ave Maria rezadia ante sus estampas y por la sola invocación de su santisimo nombre. Joannes Minguet focit A. 1765. Fig, 21.
}

11. Etpari $1897: 52$

17. Shemone, 2xigit 


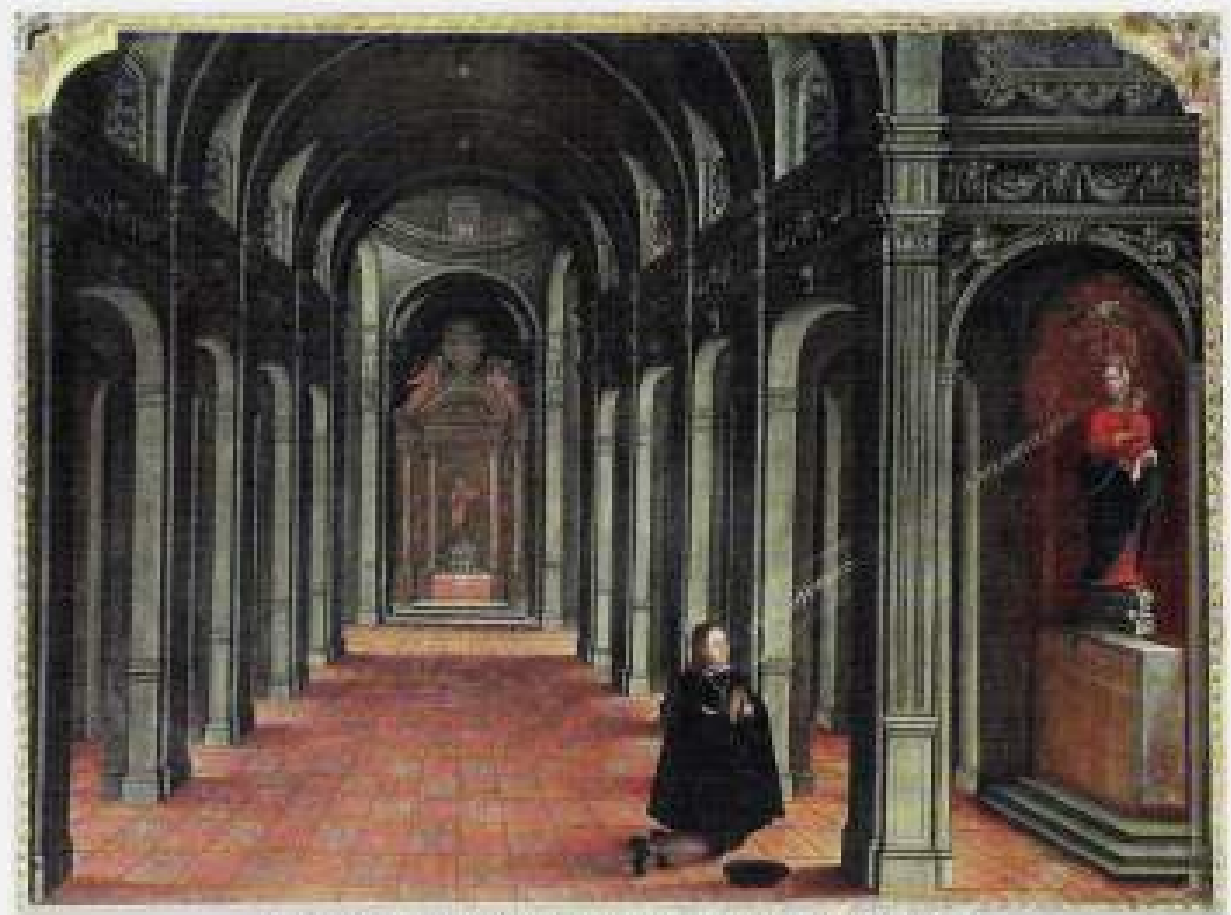

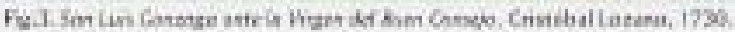

Por aquel entonces se habian trasładado los rescos de San fsidro y Sanca Maria de la Cabeza al templo jesaita y ya con la expulsión de la orden en 1767, eurante el reinado de Carlos III, cambiaria el nombre al de Colegiata de San isidro. En 1885 este recinto religioso liegó a ser Catedral, hasta 1993 en eue se teumiso la actual de La Almuderia y rolvis a ser Colegiato. Actualmente se conserva en la Iglesia de la Colegiata de San Isidra el Real de Madrid una capilia dedicada a esta advocaciôn con una escultura del siglo XX, realizada par Felix Grandin despues de la guerro civil espanola.

La pintura de Cristobal Lazano, Lbicada en el muro izunuierdo de la capilla de San Luis Gonzaga de la ledesia de San Pedro de Lima, representa en un lienzo de gran formato al interioc del remplo donde ocurre el milagro en Madrid es 1583. (fig. 3) La arquitectura muestra una pequena iglesia de nave central con bóneda de aristas y crucero con capula, asi cono arcos laterales de medio ptinto que conducen a capillas. La omamentacién res: ponde a elementos de corte clásico del siglo XV7, con dos figuras sedentes en las enjutas de los arcos: friso de mascaroaes con paños colgantes en el arranque de tas bovedas y entre los arcos pilastras corintias con estrias. Un piso ajedrezado de baldosas trabajado en perspectiva nos conduce al altar principal, dorado, de un solo cuerpo, tres calles y frontón carvo cerrado cue soporta dos ángeles sedentes de tünica roja y palmas en las manos. flanqueando cartela con el escudo jesuita y conoan, En la homacina principal, sobre sagrario de plara, la figura de Cristo Redentor con tínica azul y manto rojo, bendiciendo, con el globo terráqueo en la mano izquierda y ea los intencóumnios laterales las esculturas de San Pedro y Sin Pablo, titulares del templo.

En primer plano, hacia la derecha del lienzo destaca el altar de la Virgen con el Niño so. bre peana de plata, ambos van coronados y visten túnica roja, ella además lleva una toca 


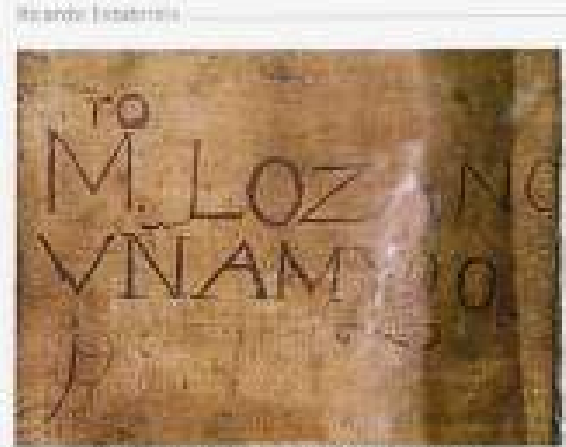

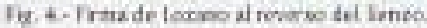

скевa, manto azal y aureola de resplandores y estrellas De la boca de la Virgen sale ans inscripeison liecha a la imversa donde es pesible leer: "HYO ENTRA, EN LA ....COMPANYA DE IHS, las palabras escritas nos coxiducen a Luis quien apatece arante, de rodillas atate el altar, vestids de nezros cons cuello blanco. en valona. y a si lado un sombrero sobre el piso.

La importancia de esta pintura para el arte thmerto del siglo XVIII es que al reverso del Eemo es posible leer: "Mto, Loxans Un amgo" (Fig. 4). Lo que nos lleva a los junceles de Cristobal Lozano, con cafacteres de paleca de sus primeros años, no solo pictónicos. sino adernis en ba forma en que firmaba por los anos treinta en cuadros que ya hemos documentado.

Antes de comentar ef tema del sizuiente lienzo sobre la vida de San Luis Gonzaga, existente eti sti capilla de fa ig esia jesuita de lima, resenaremos brevemente qué. sucedio desde

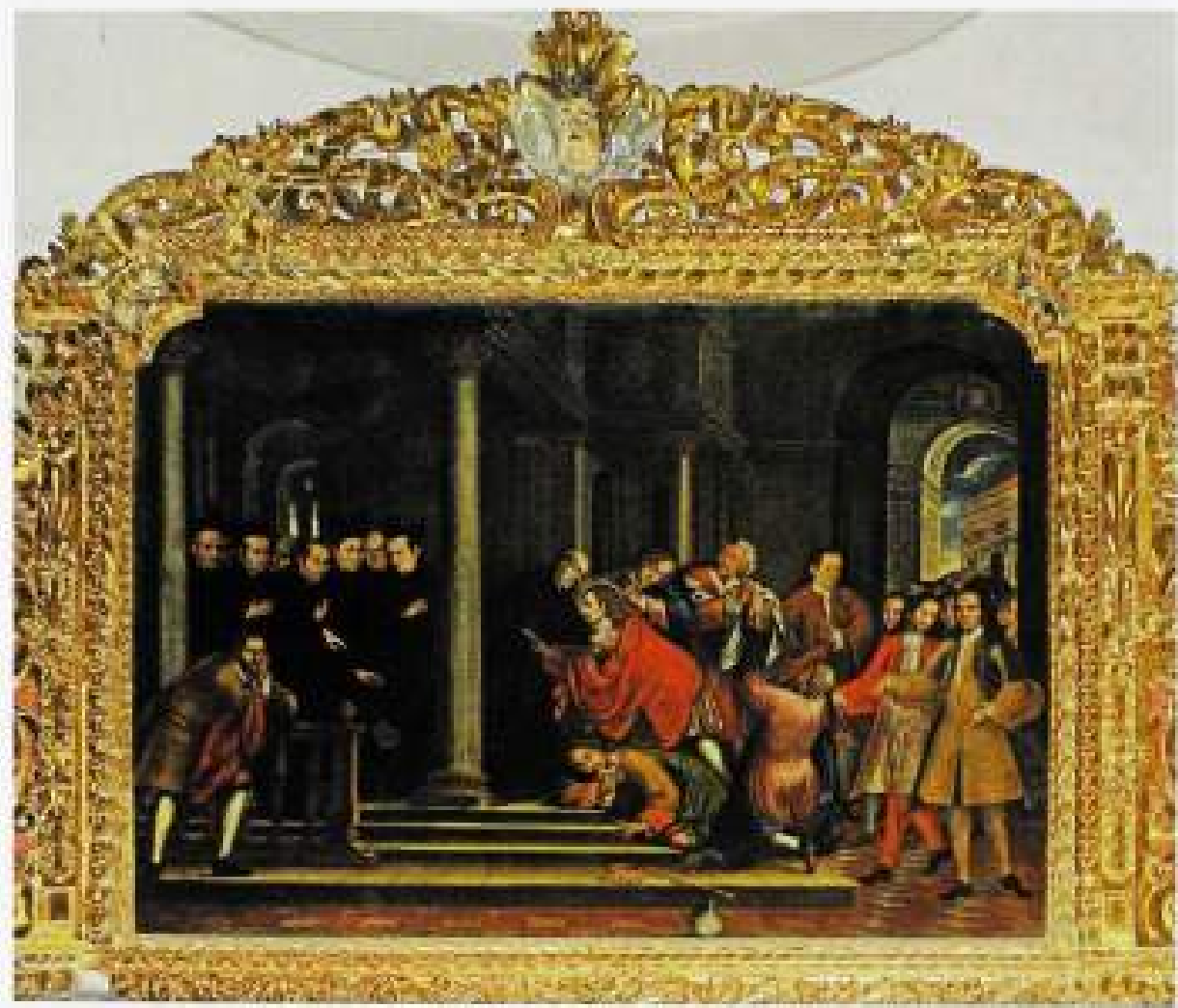

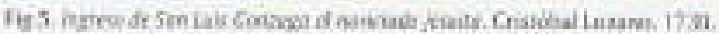


que recibio el consejo de la madre de Dios hasta ese momento. Asegurado de su woluntad a trawes de las palabras de la Virgen del Buen Consejo. Luis fue a bablar con su confesor el jesuita Feidinando Paterno para que lo zyudara a ingresar a la Compania de Jesus, el que coudiciono su ingresiy a la previa aprobación de su padre el Narqués. Al ser su perimogénito y heredero su padre se opuso rotunda. mente a tal decisión: sin embargo, al hablar con su confesor trató de disuadirlo para que no sea la ordes jesuita la elegida, sino en otra que no elimimara sus dignidades, pero no lo comencio, for aquel entonces marió el

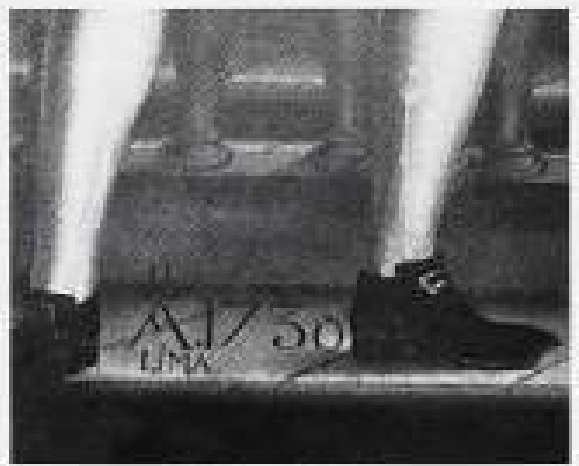

Figh Detalle de la foctus. principe 0 . Diego y Luis se via liberado de las obligaciones de palacio, su familia regres ó talia y su padre despues de muchas vicisitudes aprobo su dlecision y escribió n si ptino Scipione Gonsaga, por entonces Patriarca de Jerusalén y más adelante cardenal de la lglesia, para que de su perre hablase con el Padre General de la Compañia, el P. Chucio Aquaviva y le ofreciese asul primogénito

En el proceso, antes de ingresar a la orden, Luís tuwo que saperar atros problemas como el de contar con la aprobación del Emperador para que cediera en su hermano la sucesión de su padre el marques, para ello contó con ef certero apoyo de Doria Leowor de Austria; ducuesa de Mantua tia del Emperador Rodolfo y logro dicta jicencia cuando ya contaba con diecisiete años. Al poco bempo en Montuz, en 1585, decile hacer los Ejercicios Espirituaies, antes de cntrat a la Companita.

ta culminación de su sueto sucede en Koma después de un largo recorrido desde Mantua, visitó al P. Aquariva, a algunos cardenales y at Papa Sisto V. Firalmente, el dia 25 de noviembre de 1585, dia de Santa Catalina vigen y martir, subio a Montecavalilo donde esta el soviciado de los jesuitas acompandodo del coricjo que he habia dada su padre, Don Luis Gataneo, padre espiritual, Pedro Franciscrs del Turco su ayo, del St Scipión Gonzaga. el Dr. Juan Bautista Beno con un camarero y otros criados $^{13}$.

En una de sus composicianes más complejas de la decada de los treinta, Lozano desanchla el momento del recibimiento de l.uis Conzaga en el noviciado jesuta de Montecavillo (Fig.s. El marco escenografico muestra una arquitectura adintelada. sopertada por columnas

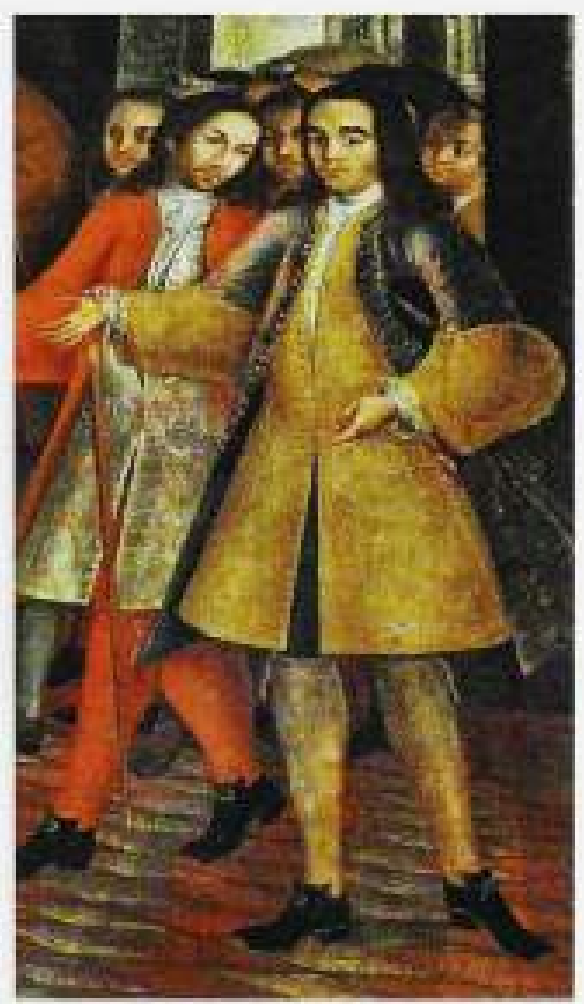

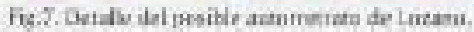




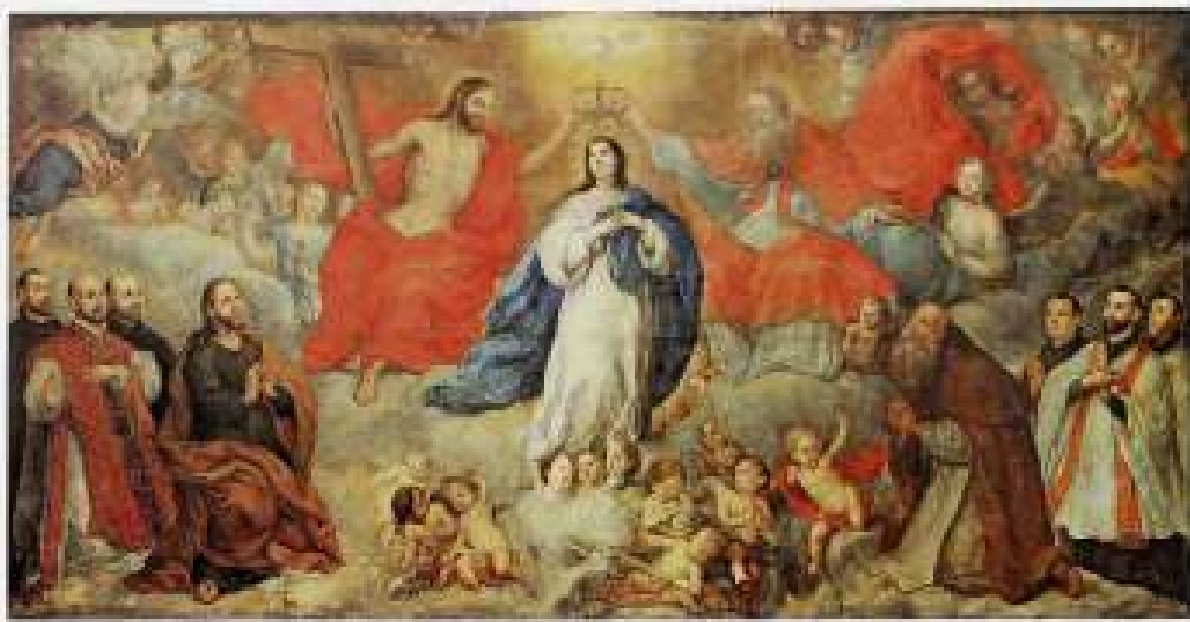

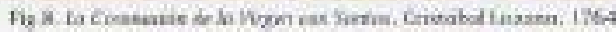

corintias, ent diversos planos sobre escalinata y dos fugas laterales en arcos continaos en fos extremos que se pienden en pequerio celaje. Contribune a la perspectwa un piso de bildosas ajedrezado. Hacia la izquierda det observador podemos apoeciar entre un par de colimmas a un grupa de jesuitas tacados, de pie, mentras que en primer plano, delante de ellos, un persinaje bico barbado recostado en un balaustre, en pose desenfidada, apoya el rostro en una mano iniestras observa la escena central. A sus pies escrito el ano de 1730 (Fig.6).

Ea la parte central del Senzo, sobre la escalinata, el joven Gonzage cubierto con ua gran manto roje que lo destaca, se inclina ante s Padre Aquaviva, quien lo recibe con un abrazo, mientras lo rodean cuatro personajes laicos en acticudes muy expresivas de devoción y lamento. Delante de ellos, solve el pisw, coronal, cerno y globo terrą̧ueo ponen en relieve las dignidades rechazadas por las de sa casa Gonzaga.

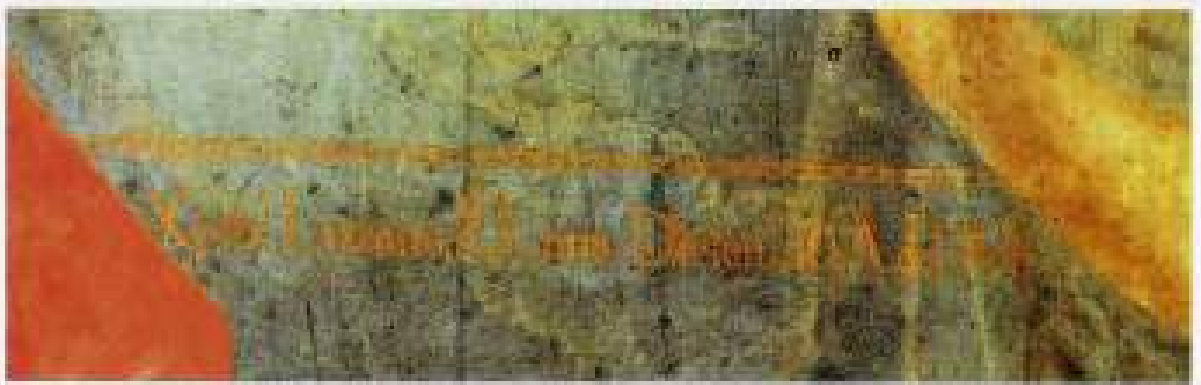

fip. Drale rie la firma viwtu

Hacia la derecha de la pintur, otro grupo de personajes delante de un arco ofisutant la atencion al boin opuesto de la escena principal para destacar la figara de uno, vestido eleganto nerite de gris y ocre, quien dirige la miradia hacia el espectadar, con los brazes levantados hacia el centro de la composicion, como presentador delacentecimiento |Fig.7| Ef excelente tatamiento pectónico de este rostro y el hecho que el personaje mire al espectador nos hace pensar ent un retrato, que bien podria corresponder al del pintor Cristibal bozano. Los caracteres flsomimicos de un mestizo hispanoamericano y la edad reflejada en su rostro, fa qaie 


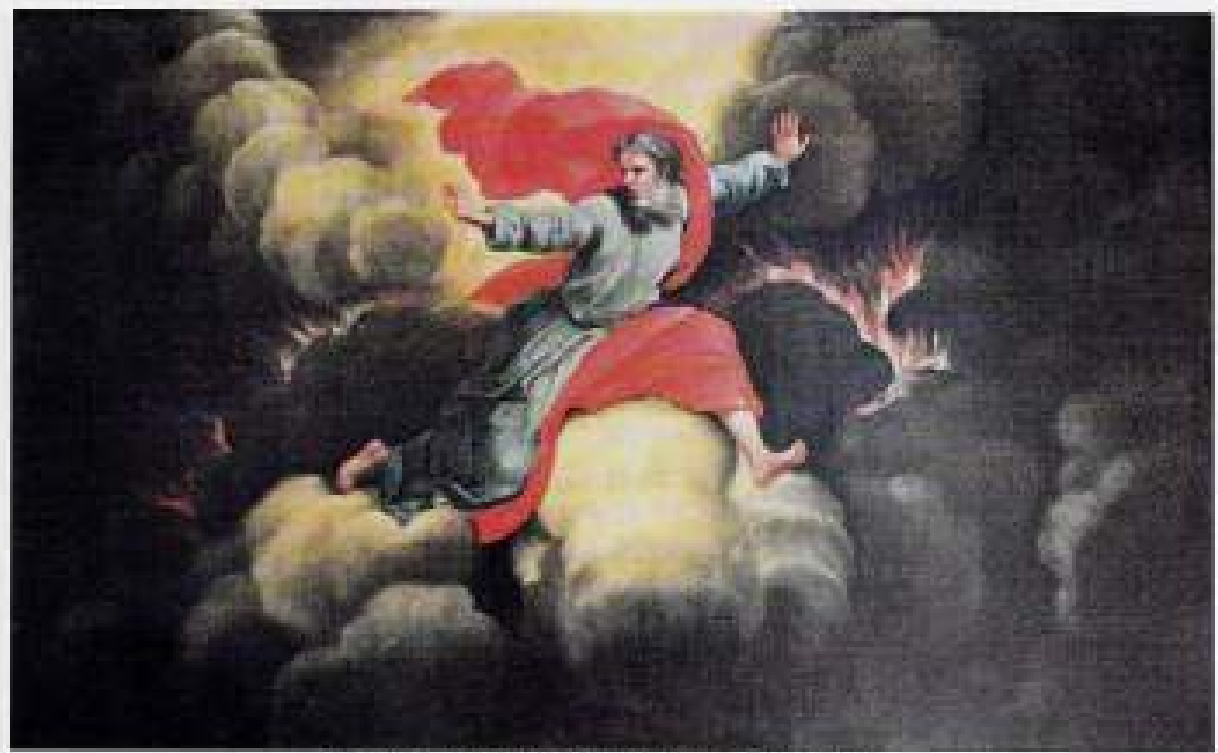

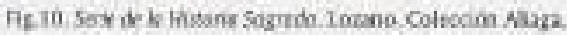

se aproxima a los treinta y un años. Jos que tendria el pintor por esa fecha, ea base al ab̆o de natalicio que hemos consignado en otra publicacion, nos ayuda a reforzat la hipótesis ${ }^{5}$.

Aparte de estas dos pinturas ineditas que sacatnos a la luz, Lozano realizo otra importan. te obra para la sacrisria de la iglesia des Seminario de San Antonio Abad de los jesuitas. ubicado en el lugar que ocupa actualmente el Centro Cultural de la Uninersidad Nacional Mayor de San Marcos, ya consignada en un trabajo anteriof " Le Conanacion de la Virzen cont Santos, una de las más importantes, nos solo por ser la de mayor formato, sino por su calidad pictórica y posque certifica ya en esta etapa de sa producción la dara influencia de Muriljo (Fig.8). El lienzo fue destinado a la iglesia de San Marcelo después de la expulsion de los jestatas y actualmente ya restatordo, se ha vbicado en el Palacio Arzobispal de lima a una altura donde es posible ver daramente la firma y la fecha exacta de su realización, sobre el globo terraqueo cue sostiene Dios Padre; 10 de diciembre de 1764 (Fig.91. Años en que Lozano ya es un piatoe consagrado en la Chidad de los Reyes, con retratos de virreyss y personejes ilustres de la ciudad y en los que se autodenominar "Profesor fnteligente ded Arte de la Pintura*, como certifica el docieneato de tasación de las pinturas de la colección de don Agustin de Salazar y Munatones en 1763. donde ubicamos muchas obras de su pincel ya estudiadas ${ }^{17}$, varias de ellas en la Coleccion de la familia Aliaga donde es posible apreciar, en una serie de la Historia Sagrada, que si bien pueden tener su fuente primera en la serie de grabados de jan Wierix (Kan de Velde 1990), la influencia evidente de la dinimica del barroco murillesco ya se hece presente en un Dios Padre wolando al viento, en pose rebuscoda con las vestiduras agitadas. (Fig. 10).

El uso elel grabado de origen flamenco, no está ausente en los cuarenta atos de producción certificada de Lozano, como hemos anotado, la vimos en varios lienzos de la colección

\footnotetext{
15. Estatiriclis: 2001: 29\%.

16. Fishraik 2001, 300.309.

17 Etahticin 2001, 3001.
} 


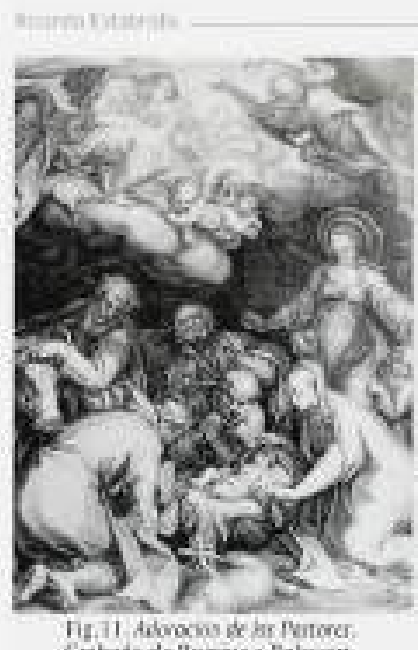

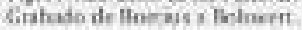

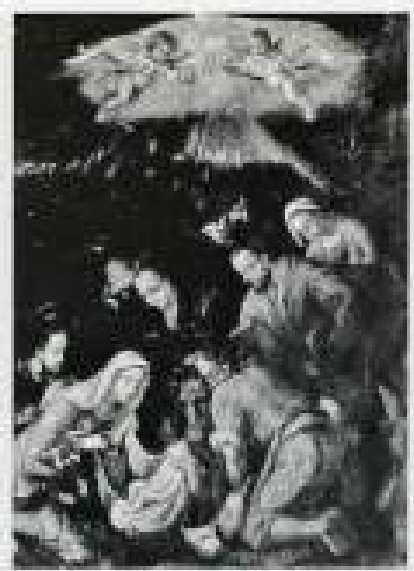

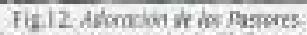
Inam. shoutuvis diff farmen

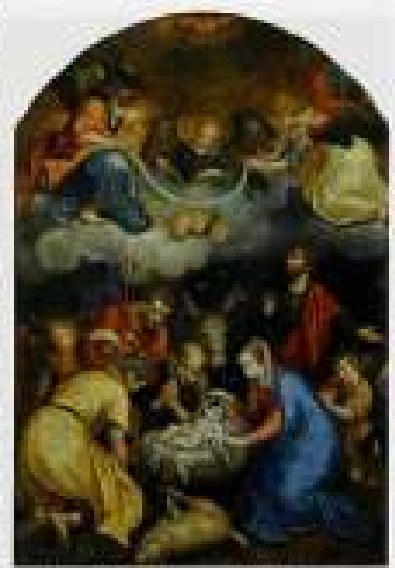

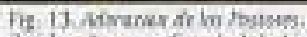
Amhadns Louns, Corodral de lima

citada, asf conto so otros de montamentos religtosos. Existe un grabado en patticular que tuso anucho éxito en Espana e influyb en el rissmo Muritlo, en México en el pántor josé Juarez y fue de la preferencis de mestro ilustre pintor, dencostrador en la cancidad de obros en que usó partes de el, sobre todo en la somposicion cie los anueles nos referimos a la estanys de la "Kdoracion de los Pastones" de Boetius a Bolswert (Fig. 111. Aparecen detalles She la misma en obras de Lozano tales como: Saita Ano educando a la Vigen, en la colecciōn Alaga o en la Apateosis de Sen Combilo, en la sacristia de la lglesta de la biena Muerte y en mactas otras, incluso desde fechas tempranas en la serie de la Vido de la Virgen del Monasterio del Carmen thig. 12 .

En la Catedral de Lima se corserva un lienzo de la Adoracivin de las Pastars, de tegular formato que es el mas fiel al grabado de Betius a Bolswert, con pequenas yarantes como el cainbio de ubicacion de Sanjose y la inclusión del asmo, Sus caracures de pincel y colorido nos permiters ralacionarlo cisn Lozano, sobre todo por la expecial preferencia para su uso demostrado en muchas de stis ohras. Creemos que el estudio minucioso de la estampa, al usarla para fa pintura de la Catedral, le permitió más adelante y con msyor facilidad utilizar. algunas de las partes adaprandolas para sus composiciones posteriotes, ajenas al tema icenografice original" (Fig. 13).

Con las obras estudiadas en este articulo, se suman ya medio centear de piaturas entre firmadas y atritudas que tenemos registridas de este artista plastico. al que consideramos el mas importante pintor limeño del sigio XVIII paradigma de la época en pincel y contex. to. el quz reclama la conservacion y puesta en valor de su legado at patrimonio culturat del Peril.

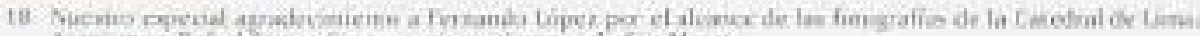

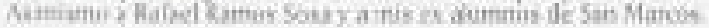




\section{BIBUOCRAFIA}

Crpari s], Virsilio

1897 Woda iz Sar Luis Ganzosa. Patring de in funcutud. New York. Finsiedeln, Benziger Editares de la Santa Sede Apostrólica.

\section{Capel, Aatonio}

1969 Lo igiesia de Sar Pedo de Lima. Lima. I lalli.

Estabridis. Rocardo

2001 Cristobal Lozano, paradigma de ls pintura limeia del siglo XVIII. En: Bowroco Weraomericana fowitanis, Atre, Espacioy Sonedad. Universidad Pablo de Olsvide (ed.): 345-364. Sevila, Edicianes Giraida,

Pinacoteca de Palacio de Gobiemo In fef Comerio del 22 de abril de $1984 \mathrm{p}$. Cl.

Cristobbal Lozano en el Monasterio del Carmen En El Converio Lima 8 de marzo de 1994. D. C?

E retrato en Lima en el siglo XVII: tas pinturas de Cristóbal Lozano

2004 En: Tradicion, estiob escucio en lo pintura ibrromencricens. Segles XVJ.XWIr México BCP, Banamex. Al OE y el IEE de la UNAM.

\section{Ilart-terrí, Emilio}

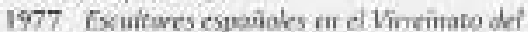
Peri, I84, Lima huan Mejía Baca.

Hecter Schenone

2008 fcanozrofin del Art Colonial Sagte Maria. P317. Boenos Aires. Edic. Universtdad Católica.

\section{Kesunoki. Ricardo}

2010 "La reina de las artes" pibturay cultura leirdods en Lima 11750-18000. En Marta tukukin. Illopa 7, 51-61, Lima. Kevista del Institato de lomestieacianes Museulogicas y Arristicis de la Unnorsidad Racando Palna:

\section{San Cristóbul, Antonio}

2005 B Barroco Vimeinal Fenaano. En ellil Coagresa Internacionda/Sevilla, octubre del 2001). En Atrio Ja/n - 149. Rensto fie Histone do Arte, Sevillat Uniwersidad Pabla de Olaride.

\section{Scastuy. Francisco}

1999 Temas discicos en el arte colonial trispanogachericans. En La tradickil cidisan m el Peni Wirrino, Lima Liniversidad nacional Maryor de San Maroas.

Vas de Velde, Carl

1990 fon Werix. The creotion ond the corle history of $\operatorname{ann} 1607-1605$ Londea. Richand i Fegen \& Co

Vargas Uẹarte, Rubén

1956 C.a lekesia de $5 a n$ Reuro de Lima. Lima.

Wuffarden. Lais Fcluardo

2004 La Catedral de Limay el triunto de la pintura En la Basica Carntrar de Lima 241-317. Lima. Banco de Credito del Peri. 